\title{
Didier Luciani, André Wénin (dir.), Le pouvoir. Enquêtes dans l'un et l'autre Testament
}

Paris, Éditions du Cerf, coll. « Lectio Divina », 2012, 384 p.

\section{Ronan Teyssier}

\section{(Q) OpenEdition}

\section{Journals}

Édition électronique

URL : http://journals.openedition.org/assr/24651

DOI : $10.4000 /$ assr.24651

ISSN : $1777-5825$

Éditeur

Éditions de l'EHESS

Édition imprimée

Date de publication : 30 décembre 2012

Pagination : 231

ISSN : 0335-5985

Référence électronique

Ronan Teyssier, «Didier Luciani, André Wénin (dir.), Le pouvoir. Enquêtes dans l'un et l'autre Testament », Archives de sciences sociales des religions [En ligne], 160 | octobre-décembre 2012, mis en ligne le 18 mars 2013, consulté le 21 septembre 2020. URL : http://journals.openedition.org/assr/24651 ; DOI : https://doi.org/10.4000/assr.24651

Ce document a été généré automatiquement le 21 septembre 2020.

(c) Archives de sciences sociales des religions 


\section{Didier Luciani, André Wénin (dir.), Le pouvoir. Enquêtes dans l'un et l'autre Testament}

Paris, Éditions du Cerf, coll. « Lectio Divina », 2012, 384 p.

\section{Ronan Teyssier}

\section{RÉFÉRENCE}

Didier Luciani, André Wénin (dir.), Le pouvoir. Enquêtes dans l'un et l'autre Testament, Paris, Éditions du Cerf, coll. « Lectio Divina », 2012, 384 p. 
1 L'ouvrage dont il est ici question est une œuvre collective résultant des travaux du séminaire de troisième cycle en exégèse de la faculté de théologie de l'Université catholique de Louvain. Il fait suite à deux publications réalisées dans des conditions similaires sur « la Loi » et « la maison de Dieu ». Le volume dont nous discutons le contenu rassemble des contributions de D. LUCIANI la direction de

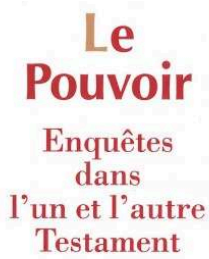
professeurs de théologie autour de la problématique du pouvoir dans un ou plusieurs livres de la Bible. Respectant l'organisation séquentielle biblique, l'ouvrage propose ainsi successivement des réflexions sur le pouvoir dans le Lévitique, le Deutéronome, le premier livre de Samuel, les livres d'Amos, d'Isaïe, des Proverbes et d'Esther, puis dans les règles de la communauté et le document de Damas, l'Évangile selon Luc, les Actes des Apôtres, les épîtres de Paul (aux Romains, à Timothée - les deux épîtres - et à Tite) et finalement dans l'Apocalypse de Jean.

2 Avant d'aborder le contenu du livre proprement dit, il convient de s'arrêter un instant sur ses objectifs. Dans l'introduction, Didier Luciani prévient le lecteur qu'il ne doit s'attendre à trouver ni présentation exhaustive du concept de pouvoir dans la Bible, ni typologie des formes que celui-ci y revêt (p. 8). Le volume propose plutôt un " parcours » sélectif dans un nombre suffisamment varié de livres de la Bible (les douze contributions couvrent sept livres de l'Ancien Testament et sept livres du Nouveau Testament) pour que le lecteur entrevoie « quelles figures du pouvoir (humain et divin, civil et religieux, spirituel et temporel) l'Ancien et le Nouveau Testament mettent en scène et surtout, quels discours ils tiennent sur la manière de l'exercer » (p. 8).

l'éclectisme caractérise indéniablement cet ouvrage, Luciani expose néanmoins très clairement le lien qui unit l'ensemble des contributions et que nous tenons pour être la thèse générale de ce volume. Selon lui, les contributeurs montrent tous à leur manière que le pouvoir n'est nullement sacralisé dans la Bible et que les auteurs bibliques prennent toujours soin de mettre en avant les garde-fous inhérents à tout type de pouvoir (p. 21).

Chacune des contributions de cet ouvrage comprend en arrière-plan un ou plusieurs débats théologiques. Plutôt que de les discuter dans le détail, nous nous bornons ici à esquisser la manière dont les multiples formes de représentation du pouvoir dans la Bible sont explorées. Pour ce faire, nous ne suivons pas la présentation séquentielle retenue dans l'ouvrage, mais optons pour une organisation thématique en trois catégories déterminées à partir du traitement que les chapitres font du pouvoir. Pour distinctes que soient ces catégories, elles entretiennent néanmoins des liens certains qui donnent à l'ouvrage sa forte cohérence interne.

5 La première catégorie rassemble les contributions qui abordent le pouvoir dans la Bible sous l'angle institutionnel en en présentant les principaux détenteurs (Dieu, ses représentants, des autorités civiles, le Diable ou encore certains éléments du peuple) et leur capacité respective d'action. Nous voyons par exemple dans la première contribution, qui porte sur le pouvoir dans le Code de Sainteté (Lévitique 17-26), que le 
pouvoir tend à être dilué ou tout au moins partagé entre Dieu et deux autres détenteurs que sont la famille et l'assemblée (p. 40). Dans la contribution suivante, Jean-Marie Carrière formule un constat similaire en ce que son analyse d'un passage du Deutéronome l'amène à évoquer l'image d'un pouvoir largement partagé qui serait «le pouvoir de tout un chacun» (p.54-55). D'autres contributions nous informent sur le contenu en termes de pouvoir, c'est-à-dire de capacité d'action, de différentes positions institutionnelles que ce soit à Qumrân (pouvoir du Mebaqqer, du Paqîd ou encore du Maskîl, p. 221-230) ou dans l'Église primitive. Traitant du pouvoir dans les épîtres pastorales (Timothée 1 et 2 et Tite), Michel Gourgues distingue en effet dans l'Église primitive le pouvoir de l'apôtre - pouvoir d'origine transcendante, c'est le pouvoir de Paul - (p. 295-302) ; celui des délégués de l'apôtre - Timothée et Tite en l'occurrence (p. 302-308) et celui de divers ministères comme l'épiscope, les diacres et les presbytres (p. 309). Enfin, deux contributions présentent plus directement le contenu du pouvoir divin en le contrastant avec d'autres pouvoirs. Dans le premier cas, Nathalie Siffer s'intéresse au pouvoir dans l'œuvre lucanienne (Évangile de Luc et Actes des Apôtres) et oppose le pouvoir de Dieu à celui des autorités terrestres persécutrices des chrétiens (p. 235-237). Dans le second cas, Jacques Descreux détaille à partir de l'Apocalypse de Jean le contenu du pouvoir créateur et bienveillant de Dieu (p. 338-341, p. 349-350) qu'il oppose au pouvoir destructeur et malveillant du Diable (p. 327-328).

6 Si les contributions de la première catégorie décrivent le pouvoir d'acteurs majeurs de la Bible, la deuxième catégorie que nous proposons rassemble les chapitres portant sur diverses facettes de la contestation du pouvoir. On y trouve tout d'abord des contributions sur la posture prophétique face aux pouvoirs économique (Amos) et politique (Isaïe). Le prophète est dans cette perspective un acteur dont le rôle est de rappeler constamment que « toute action politique doit être fondée sur la confiance en YHWH et sur la crainte de sa colère» (p. 162). Le même message ressort de l'étude de Maurice Gilbert sur la vision du pouvoir dans un passage du livre des Proverbes.

7 On trouve également dans cette catégorie deux contributions discutant d'attitudes individuelles face au pouvoir civil. Il s'agit des excellents chapitres de Jean-Daniel Macchi sur les modalités possibles de la résistance face au pouvoir tyrannique dans le livre d'Esther (le refus, la ruse ou la force) et de Jean-Noël Aletti sur le passage très célèbre de l'épître paulinienne aux Romains (Romains 13, 1-7) dans laquelle est traitée la question de la soumission due aux autorités civiles.

8 La troisième catégorie comporte uniquement la contribution d'André Wénin sur l'acquisition et la transmission du pouvoir dans le premier livre de Samuel (la transition du pouvoir de Samuel à Saül). L'auteur montre à travers le récit de la fin de la « judicature » de Samuel comment ce dernier s'accroche au pouvoir et il illustre ainsi parfaitement par l'exemple le fait que le pouvoir tient en général ses titulaires aussi sûrement que ceux-ci le détiennent (p. 64-65). Parce qu'il présente un cas concret, Wénin complète fort utilement les contributions de la première catégorie qui traitent du pouvoir de manière théorique. Parce qu'il aborde la problématique de la transmission du pouvoir, cette contribution devrait à l'instar des chapitres de la deuxième catégorie intéresser au plus haut point les spécialistes de science politique.

9 Sans que nous nous prononcions sur la valeur théologique des contributions de cet ouvrage, il nous semble que ce dernier atteint sans conteste son premier objectif de donner à voir la diversité des représentations du pouvoir dans la Bible. L'éclectisme des 
textes choisis, des situations considérées et des méthodes d'analyse employées offre un aperçu sinon exhaustif du moins fort complet de la question du pouvoir dans la Bible.

L'ouvrage atteint également son second objectif en démontrant clairement que le pouvoir est toujours partagé et limité dans la Bible. Il nous paraît même possible de dire sur ce dernier point qu'aucun personnage biblique ne semble être omnipotent ; pas même Dieu, dont le pouvoir apparaît comme étant également limité. L'ouvrage arrive donc à offrir une image du pouvoir dans la Bible qui partage de nombreux points communs avec les conceptions modernes comme la séparation des pouvoirs et l'équilibre entre ces derniers.

11 Ce volume présente une riche mosaïque d'études qui renvoient collectivement une image non sacralisée du pouvoir. Par la diversité de son propos et son message distancié, ce livre est une remarquable contribution à l'étude du pouvoir dans la Bible et même du pouvoir de manière générale. Notre seul regret est de relever que dans la plupart des chapitres le concept de pouvoir ne fait pas l'objet d'un effort de définition explicite très poussé. Seules les contributions de Gourgues sur les épîtres pauliniennes à Timothée et Tite (p. 292, p. 322) et de Descreux sur l'Apocalypse de Jean (p. 326) échappent à cette remarque.

Cela étant dit, la qualité d'ensemble de l'ouvrage et le fait qu'il s'agisse du troisième livre du genre résultant du séminaire en exégèse de la faculté de théologie de l'Université catholique de Louvain, nous paraissent légitimement garantir son succès dans les cercles théologiques. Nous souhaitons également que cet opus, ou à tout le moins certaines de ses contributions (par exemple celles de Wénin, Macchi ou encore Aletti), puissent trouver l'écho qu'il nous semble mériter auprès des spécialistes d'anthropologie politique, d'histoire des idées politiques et de philosophie politique. Il ne fait aucun doute qu'il a quelque chose de tout à fait original et significatif à leur apporter. 